\title{
Tone Smith*
}

\section{Wie radikal ist der Green New Deal?}

Zusammenfassung: Die Idee eines Green New Deal (GND) ist nicht neu, aber in den letzten Jahren wurde eine Reihe von - mehr oder weniger radikalen - Vorschlägen entwickelt, insbesondere von linken Gruppen. Der Beitrag analysiert vier GND-Vorschläge und fragt, ob sie radikal genug sind, um eine Grundlage für die notwendige sozial-ökologische Transformation zu legen. Obwohl die Vorschläge viele progressive und radikale Elemente aufweisen, kommt der Beitrag zu dem Schluss, dass sie nicht weit genug gehen. Das Hauptproblem ist, dass ein New Deal seinem Wesen nach ein wirtschaftliches Wachstumsprojekt ist und damit den Mythos fördert, dass ein grünes Wachstum möglich ist.

Stichwörter: Ökologische Ökonomie, grünes Wachstum, Degrowth, Arbeit, imperiale Lebensweise

\section{How radical is the Green New Deal?}

Abstract: The idea of a Green New Deal (GND) is not new, but the last couple of years have seen the development of a range of - more or less radical - proposals, especially from left-wing groups. This article analyses four GND proposals and ask whether they are radical enough to provide a basis for the needed social-ecological transformation. Although there are many progressive and radical elements in these proposals, the article concludes that they do not go far enough. The main problem is that a New Deal is by its very nature an economic growth-project, and hence furthers the myth of the possibility of green growth.

Keywords: Ecological economics, green growth, degrowth, work, imperial mode of living

* Tone Smith ist Umweltökonomin und Humangeografin; sie arbeitet als freiberufliche Autorin. 


\section{Einleitung}

D erzeit kursieren eine Reihe von Vorschlägen für einen Green New Deal (GND) von grünen Parteien, Gruppen innerhalb linker Parteien, Think Tanks und sozialen Netzwerken. Trotz einiger Unterschiede beschäftigen sie sich alle mit der aktuellen, ineinander verflochtenen sozialen, ökologischen und ökonomischen Krise. Sie konzentrieren sich insbesondere auf den Übergang von einer auf fossilen Brennstoffen zu einer auf erneuerbaren Energien basierenden Wirtschaft und auf die Schaffung von Arbeitsplätzen.

Die Idee eines GND wurde bereits in den 1990er Jahren von einer Koalition aus deutschen Grünen und Sozialdemokrat:innen diskutiert (Candeias 2013), und der erste Vorschlag für einen GND wurde 2008 von einer britischen Gruppe veröffentlicht, die sich The Green New Deal Group nannte. Doch erst im Februar 2019, nachdem die US-demokratische Kongressabgeordnete Alexandria Ocasio-Cortez und der US-demokratische Senator Ed Markey eine neue Resolution für ein Green-New-Deal-Gesetz vorlegten, gewann das Thema zunehmend öffentliche Aufmerksamkeit. Seitdem haben eine Reihe progressiver Gruppen innerhalb linker Parteien ihre eigenen GNDs initiiert. Diese neueren Vorschläge haben einen breiteren sozialen Fokus, der über die Schaffung von Arbeitsplätzen hinausgeht und Anliegen wie Gerechtigkeit und Ungleichheit sowie die Anliegen marginalisierter Bevölkerungsgruppen aufgreift.

Im Kern baut die Idee eines GND auf Roosevelts umfangreichem Programm zur Lösung jener sozialen und wirtschaftlichen Probleme auf, die im Zuge der Großen Depression in den 1930er Jahren entstanden. Roosevelts New Deal bestand darin, die Wirtschaft anzukurbeln, indem er gleichzeitig den Arbeitslosen Arbeitsplätze und damit Einkommen verschaffte und die Wirtschaft mit Nachfrage (bzw. Konsum) versorgte, um die Rezession zu überwinden. Es war ein Projekt, das auf der Wirtschaftstheorie von Keynes basierte und die Notwendigkeit betonte, die Nachfrageseite der Wirtschaft zu stimulieren. Die heutigen Vorschläge für einen GND zielen nicht mehr allein darauf ab, die Wirtschaft wieder in Gang zu bringen, indem mehr Arbeitsplätze geschaffen werden, sondern sie wollen dieses Ziel darüber hinaus mit einem Umbau der Wirtschaft verbinden, der sich an den zentralen ökologischen Herausforderungen orientiert, vor denen die Welt derzeit steht - insbesondere die Klimakrise.

Angesichts der Popularität verschiedener Ansätze eines GND zur Bewältigung der Herausforderungen unserer Zeit lohnt es sich, diese genauer unter die Lupe zu nehmen. Aus einer progressiven, rot-grünen Perspektive ist es wichtig zu beurteilen, inwieweit sie mit Vorschlägen für eine radikale sozialökologische Transformation übereinstimmen. In diesem Artikel werde ich daher insbesondere untersuchen, wie die Vorschläge die Probleme des Wirt- 
schaftswachstums aus ökologischer Perspektive anvisieren, und wie sie mit dem Problem umgehen, dass der reiche globale Norden seinen unverhältnismäßig hohen Anteil am globalen Konsum von Ressourcen und Umweltsenken reduzieren muss - was auch als »imperiale Lebensweise« beschrieben wurde (Brand/Wissen 2017). Die Analyse orientiert sich hauptsächlich an der Theorie der sozial-ökologischen Ökonomie, ergänzt durch breitere Forschung zu sozial-ökologischer Transformation (Brand 2014).

Der Artikel beleuchtet zunächst eine Auswahl von Vorschlägen des GND. Anschließend stelle ich eine biophysikalische Perspektive auf die Wirtschaft vor, die in der ökologischen Ökonomie begründet ist und als Grundlage für die Diskussion der GND-Vorschläge dient. Die Analyse konzentriert sich auf den Inhalt der verschiedenen Vorschläge, geht aber nicht auf Fragen der politischen Machbarkeit oder der politischen Strategie ein. ${ }^{1}$ Abschließend stelle ich dar, inwiefern die Bewertung der GND-Vorschläge auch für die aktuellen Debatten über die Ausgestaltung der Konjunkturpakete relevant ist, die für eine wirtschaftliche Erholung nach der Corona-Krise sorgen sollen. Insgesamt argumentiere ich, dass die GND-Vorschläge einige zentrale Themen für eine sozial-ökologische Transformation ausklammern. Gleichzeitig enthalten sie jedoch auch viele Elemente, die Teil einer solchen Transformation sein könnten.

\section{Einige ausgewählte Vorschläge für einen Green New Deal}

In der folgenden Darstellung von vier ausgewählten Vorschlägen für einen GND sollen diese nicht im Detail wiedergegeben werden, vielmehr möchte ich Teile der Debatten über einen GND nachzeichnen wie auch einige der progressiveren Varianten präsentieren. Der Bericht der Green New Deal Group aus dem Jahr 2008 stellt den ersten Vorschlag dar, und wohl auch jenen mit der strengsten keynesianischen Grundlage. Der Vorschlag der USDemokrat:innen hat wiederum international viel Medienaufmerksamkeit erhalten. Die Vorschläge aus der britischen Labour-Partei (Labour for a Green New Deal) und von der Bewegung DieM25 zeichnen sich durch ihre vergleichsweise radikalen Elemente aus.

\section{Green New Deal Group (Großbritannien, 2008)}

Die Green New Deal Group (im Folgenden GND-Gruppe) bestand zu Beginn aus neun Mitgliedern mit Verbindungen zu NGOs, zur britischen Zeitung The Guardian, zu Think Tanks, Forschung und Politik (z. B. zu einem Parlaments-

1 Das bedeutet, dass ich auch keine Analyse des Biden-Plans, also Bidens moderaterer Version des GND, im Vergleich zum Original vornehme. 
mitglied der Grünen Partei). Sie hat sich im Laufe der Jahre leicht verändert und umfasst nun auch einen Labour-Abgeordneten. Die Gruppe begann ihre Arbeit im Jahr 2007, als sich bereits erste Krisentendenzen abzeichneten: niedrige Wachstumsraten, Arbeitslosigkeit und ökologische Herausforderungen. Als die Gruppe im Juni 2008, also noch vor dem großen Crash (Lehman Brother), ihren ersten Bericht veröffentlichte, warnte sie bereits, dass wir auf eine wirtschaftliche Depression zusteuern, wie wir sie seit der Großen Depression nicht mehr erlebt haben (The Green New Deal Group 2008). In dem Bericht identifizierte die Gruppe eine Dreifachkrise, bestehend aus einer Finanz-, Klima- und Energiekrise (Peak Oil). Die umfassende Deregulierung der Weltwirtschaft, die in den 1980er Jahren begann, gilt als wesentliche Ursache für diese Situation.

Vor diesem Hintergrund suchte die Gruppe nach Maßnahmen, um die Wirtschaft wieder zum Wachsen zu bringen, Arbeitsplätze zu schaffen und gleichzeitig auf die Umwelt Rücksicht zu nehmen (in der praktischen Umsetzung eigentlich nur den Klimawandel). Dazu schlägt sie vor, auf die historischen Lehren von Roosevelts New Deal und der Mobilisierung für den Zweiten Weltkrieg zurückzugreifen. Da die Gruppe in der Deregulierung des globalen Finanzsektors den entscheidenden Grund für die Entstehung der Finanzkrise sieht, setzt sie vor allem auf die Re-Regulierung dieses Sektors, wobei zugleich die Nutzung fossiler Brennstoffe reduziert sowie der Arbeitslosigkeit und dem Nachfragerückgang entgegengewirkt werden sollen. Im Hinblick auf Klimapolitik stützt sich der Bericht stark auf den Stern-Bericht zur Ökonomie des Klimawandels und das darin dargelegte Kosten-Nutzen-Denken, auf dessen Grundlage der Bericht zu dem berühmten Fazit kommt, dass es nur etwa ein Prozent des BIP pro Jahr kosten wird, die schlimmsten Auswirkungen des Klimawandels zu vermeiden (Stern u.a. 2006).

Insgesamt waren die wichtigsten politischen Empfehlungen, die aus dem Bericht von 2008 hervorgingen: i) die strukturelle Umgestaltung der Regulierung nationaler und internationaler Finanzsysteme und eine grundlegende Neugestaltung der Steuersysteme, ii) die Forderung nach einem nachhaltigen Programm für Investitionen in Energieeinsparung sowie erneuerbare Energien, sowie iii) die Kopplung dieser Maßnahmen an eine traditionell keynesianische Politik der Nachfragesteuerung.

\section{US-Demokraten (2019)}

Am 7. Februar 2019 reichten Alexandria Ocasio-Cortez und Senator Ed Markey eine Resolution ein: den Gesetzentwurf für einen Green New Deal (jetzt House Resolution 109). Die Resolution wurde von mehr als 70 demokratischen Kongressmitgliedern unterzeichnet und erlangte sowohl national als auch 
international große Aufmerksamkeit. Die Resolution nennt eine Reihe von Gründen für Reformen und entwirft eine umfassende Vision, wie das Land im nächsten Jahrzehnt gleichzeitig die Ungleichheit bekämpfen, den Klimawandel angehen, gut bezahlte Arbeitsplätze schaffen und besonders schutzbedürftige Bevölkerungsgruppen unterstützen kann.

Konkret stellt die Resolution einen Zehnjahresplan vor, um zu einer Netto-Null-Emissionen-Wirtschaft zu gelangen. Erreicht werden soll dies unter anderem durch einen Umstieg auf erneuerbare Energien und den Ausbau des öffentlichen Nahverkehrs. Die Resolution deckt jedoch auch eine Reihe anderer Umweltbereiche ab und zielt darauf, den Zugang zu sauberer Luft und sauberem Wasser, zu gesunder Nahrung und zu einer nachhaltigen Umwelt für alle Amerikaner:innen zu sichern, wobei auch gefährliche Mülldeponien beseitigt werden sollen. Die Resolution äußert sich jedoch nicht zur Rolle der Kernenergie oder von Kohlenstoffabscheidung und -speicherung (CSS).

In sozioökonomischer Hinsicht fordert die Resolution einen garantierten Arbeitsplatz mit fairer Entlohnung, aber auch Familien- und Krankheitsurlaub, bezahlten Urlaub und Rentensicherheit. Hinzu kommen eine universelle, qualitativ hochwertige Gesundheitsversorgung, kostenlose Hochschulbildung und der Zugang zu bezahlbarem, sicherem und angemessenem Wohnraum. Obwohl diese Vorschläge im US-Kontext bereits radikal und ehrgeizig sind, sticht insbesondere ihr Fokus auf Gerechtigkeit hervor. Entwickelt in engem Kontakt mit den Bewegungen für Umweltgerechtigkeit (The Sunrise Movement) fordert die Resolution den Schutz derjenigen Gruppen, die bereits heute besonders mit Umweltzerstörung konfrontiert sind, einschließlich der People of Colour sowie armer und indigener Bevölkerungsgruppen und Gemeinden.

\section{Labour for a Green New Deal (Großbritannien, 2019)}

Kurz nach der Veröffentlichung der US-Resolution startete eine Gruppe britischer Labour-Mitglieder - inspiriert von den US-GND - eine Graswurzelkampagne, um die Partei zur Verabschiedung eines radikalen und sozialistischen Green New Deal zu drängen, und zwar »mit dem Ziel, die britische Wirtschaft zu transformieren, Ungleichheit zu bekämpfen und die eskalierende Klimakrise zu bewältigen $\aleph^{2}$.

Wie der US-Vorschlag zeichnet sich auch der britische Labour-GND durch einen starken Fokus auf Gerechtigkeit aus. Allerdings konzentriert er sich stärker auf einen gerechten Übergang (just transition), insofern als er einen besonderen Schwerpunkt darauf legt, neue Arbeitsplätze für diejenigen zu

2 »Labour for a Green New Deal«, https://www.labourgnd.uk/, Zitate im Folgenden nach eigener Übersetzung. 
schaffen, deren Jobs im Übergang wegfallen. Der Labour-GND hat auch ein explizit sozialistisches Profil, indem er den Klimawandel von vornherein als eine Klassenfrage begreift: »Beim Klimawandel geht es grundlegend um Klasse, denn er bedeutet Chaos für die Vielen, während die Wenigen profitieren « (Taylor 2019, eig. Übersetzung). Damit wird die Arbeiter:innenbewegung an die Spitze der grünen Transformation gestellt. Insgesamt fordert die Kampagne eine auf einzelne Regionen zugeschnittene Garantie für grüne Arbeitsplätze, die Ausweitung des öffentlichen Eigentums und der demokratischen Kontrolle über eine Reihe von Wirtschaftssektoren sowie massive Investitionen in öffentliche Infrastruktur und erneuerbare Energien.

In Bezug auf den Klimawandel fordert der Labour-GND (absolute) NullKohlenstoff-Emissionen bis 2030. Um dies zu erreichen, plädiert er für einen schnellen Ausstieg aus allen fossilen Brennstoffen in Kombination mit groß angelegten Investitionen in erneuerbare Energien. Darüber hinaus wird mit einem grünen, integrierten öffentlichen Verkehrsplan ein völlig neues Verkehrssystem anvisiert, das Probleme der Ungleichheit, der öffentlichen Gesundheit und der Klimakrise in ihrer Verwobenheit angehen soll. In diesen Sektoren sollen neue Arbeitsplätze entstehen, während Arbeitsplätze in den auslaufenden Wirtschaftsbereichen wegfallen.

Um einen gerechten Übergang zu erreichen, wird die Forderung nach "gut bezahlten, gewerkschaftlich organisierten grünen Arbeitsplätzen« in Kombination mit der Sicherung der Grundrechte aller Menschen durch Sicherstellung der allgemeinen Grundversorgung (universal basic services, UBS) erhoben. Letzteres gilt als zentral für die notwendige wirtschaftliche Transformation und für den Aufbau einer neuen sozialistischen Wirtschaft, in der »die Grundbedürfnisse jeder:s einzelnen befriedigt sein sollen, damit er:sie sich entfalten kann«. Die allgemeine Grundversorgung soll folgende Bereiche abdecken: Bildung, Gesundheit und Soziales, Energie, Wasser, Wohnen, Verkehr, Informationstechnologie und Lebensmittel. Eng verbunden mit der Sicherstellung der allgemeinen Grundversorgung ist das Ziel, öffentliches und demokratisches Eigentum auszuweiten, auch in den Bereichen Verkehr und Energie. Schließlich enthält der Deal auch ein internationales Element. Entwicklungsländer sollen bei der Klimawende unterstützt, Klimaflüchtlinge aufgenommen und zugleich Vertreibung verhindert werden.

Auf dem Labour-Parteitag im September 2019 wurde ein Antrag zum GND beschlossen ${ }^{3}$, wobei jedoch mehrere der ursprünglichen Vorschläge verändert oder verwässert wurden. Während viele der ehrgeizigen sozioökonomischen Ziele beibehalten wurden, fallen die Ziele im Umweltbereich nun schwächer

3 »Agreed conference motion«, https://www.labourgnd.uk/agreed-conference-motion. 
aus. Vor allem wurde das ehrgeizige Ziel von Null-Kohlenstoff-Emissionen bis 2030 auf das Mainstream-Ziel von Netto-Null-Emissionen geändert. Außerdem wurde das Ziel, aus allen fossilen Brennstoffen auszusteigen, durch ein Verbot von Fracking ersetzt. In der folgenden Analyse wird die ursprüngliche Version Labour for a GND bewertet.

\section{DiEM25 (Europa, 2019)}

Das Democracy in Europe Movement 2025 (im Folgenden DiEM), das 2016 vom ehemaligen griechischen Finanzminister Yanis Varoufakis gegründet wurde, beschreibt sich selbst als eine paneuropäische progressive Bewegung. Laut ihrer Website ${ }^{4}$ zielt DiEM darauf ab, »die EU zu demokratisieren, bevor sie sich auflöst«. Um dieses Ziel zu erreichen, spielt die im April 2019 gestartete Kampagne Green New Deal for Europe und ihr wegweisender Bericht Roadmap für Europas sozial-ökologische Wende (Adler u.a. 2019) eine zentrale Rolle. ${ }^{5}$

Die Kampagne möchte Europas Gemeinden, Gewerkschaften, Parteien und Aktivist:innen hinter einer gemeinsamen Vision von Umweltgerechtigkeit vereinen. Der Bericht soll einen umfassenden Fahrplan für eine nachhaltige Transformation der Gesellschaft in Europa entwerfen, ausgehend von der Annahme, dass Finanz-, Wirtschafts- und Ökosysteme eng miteinander verwoben sind und dass eine ökonomische Transformation für eine ökologische Transformation unerlässlich ist.

Die Initiative weist Ähnlichkeiten mit der US-amerikanischen und der Labour-Initiative auf, zum Beispiel mit Blick auf Vorschläge wie einer Arbeitsplatzgarantie oder einer allgemeinen Grundversorgung in den Bereichen Wohnen, Infrastruktur und Gesundheit. In einigen Bereichen geht sie jedoch deutlich weiter. In Bezug auf Gerechtigkeit schlägt die Roadmap vor, eine Kommission für Umweltgerechtigkeit einzurichten, d.h. ein »internationales Gremium, das dafür sorgt, dass der grüne Wandel gerecht verläuft « (Adler u.a. 2019: 10).

Die Roadmap wird in zehn Säulen übersetzt (Adler/Wargan o.J.). Eine der Säulen stellt das aktuelle Bekenntnis zum Wirtschaftswachstum in Frage und fordert "Schluss mit dem Dogma des endlosen Wirtschaftswachstums «. Konkret besteht die Forderung darin, das BIP-Wachstum als primären Maßstab für den Fortschritt aufzugeben und durch ganzheitliche Maßstäbe zu ersetzen. Die Kritik des Wirtschaftswachstums ist jedoch kein zentrales Ele-

4 Democracy in Europe Movement 2025 (DiEM25), https://diem25.org/de/.

5 DiEM hat die Kampagne initiiert, sie wird unterstützt von einem Bündnis aus 14 Organisationen. 
ment des Berichts. Dennoch weist er darauf hin, dass das vorherrschende Modell des Wirtschaftswachstums in den Ländern des globalen Nordens auf der Extraktion von Ressourcen aus dem globalen Süden basiert. Außerdem heißt es, dass der »Green New Deal für Europa keine destruktive Agenda eines ıgrünen Wachstums` fortsetzen« darf (Adler u.a. 2019: 26).

\section{Theoretische Grundlagen}

Bei der Bewertung der GND-Vorschläge werde ich mich insbesondere auf zwei Dimensionen konzentrieren: i) inwiefern die Ansätze tatsächlich realistisch sind, die Umweltkrise zu bewältigen und ii) den Aspekt der internationalen Solidarität. Um beurteilen zu können, ob die GND-Vorschläge die Wirtschafts- und Umweltkrise in einem umfassenden und transformativen Sinne betrachten, gilt es, sich die biophysikalische Dimension der Ökonomie vor Augen zu führen. Daher sollen zunächst die wichtigsten Argumente und Konzepte innerhalb der ökologischen Ökonomie skizziert werden (für einen detaillierteren Überblick vgl. Spash/Smith 2019).

Alle GND-Vorschläge zielen darauf $\mathrm{ab}$, eine auf fossilen Brennstoffen oder Kohlenstoff basierende Wirtschaft zu überwinden. Eine tragfähige Strategie für eine solche Transformation muss berücksichtigen, in welchem Ausmaß unsere modernen, industriellen Ökonomien durch fossile Energie ermöglicht werden, einschließlich des leichten und günstigen Zugangs zu großen Mengen dieser einzigartigen Energiequelle mit ihrer extrem hohen Energiedichte. Gerade der Zugang zu dieser spezifischen Energiequelle liegt dem enormen Anstieg des materiellen Wohlstands in weiten Teilen der Welt in den letzten zwei Jahrhunderten zugrunde. Diese Sichtweise widerspricht der verbreiteten Auffassung, dass der heutige Lebensstandard auf die hohen Produktivitätszuwächse zurückgeht. Jedoch ist die Arbeitsproduktivität in erster Linie gestiegen, weil wir menschliche (und tierische) Arbeit durch fossile Energie und Maschinen ersetzt haben - nicht, weil wir so effektiv und viel intelligenter arbeiten. Nichtsdestotrotz hat diese Entwicklung die Möglichkeit geschaffen, enorme Mengen an Gütern und materiellem Wohlstand zu produzieren. Das Problem ist jedoch, dass dies auch zu einer Zerstörung der natürlichen Umwelt und einem Übermaß an Treibhausgasen in der Atmosphäre geführt hat.

Wenn wir auf fossile Energieträger verzichten, können wir das heutige Niveau des Energieverbrauchs nicht aufrechterhalten, weil der Ausbau der erneuerbaren Energieerzeugung selbst bereits riesige Mengen an Energie und Rohstoffen benötigt. Die Produktion von erneuerbarer Energie hat einen geringeren Energieertrag pro Energieeinheit als fossile Brennstoffe. Zu- 
dem sind viele der Rohstoffe, die z. B. für die Herstellung der dazugehörigen Elektronik benötigt werden, nicht im Überfluss vorhanden und werden oft unter problematischen Bedingungen (meist im Globalen Süden) abgebaut.

Eine weitere wichtige Auswirkung der Umstellung auf erneuerbare Energien besteht darin, dass viele `Klimalösungen` die Probleme einfach in andere Umweltbereiche verlagern. Große Windkraftanlagen zum Beispiel belasten die lokale natürliche Umwelt vor allem durch die Bauinfrastruktur und die für ihre Wartung notwendige Straßeninfrastruktur. Sie können auch lokale Arten (insbesondere Vögel) bedrohen. Der Bau von Windrädern ist zudem nicht $\mathrm{CO}_{2}$-frei, da dieser große Mengen an Beton benötigt. Die Produktion von Biokraftstoffen ist ein weiteres Beispiel für erneuerbare Energien, die vielfach zu einer Verringerung der Biodiversität durch Abholzung und die Anlage von Plantagen geführt (und gleichzeitig die Ernährungssicherheit in vielen Ländern verringert) hat. Erneuerbare Energie, deren Generierung, Speicherung und Verteilung auf industrieller Produktion beruht, wird daher oft fälschlicherweise als ssaubere oder sgrüne` Energie bezeichnet, da jede Produktion Auswirkungen auf die Umwelt hat. Daher muss der Fokus auf der Einsparung von Energie und Rohstoffen liegen, nicht allein auf dem Wechsel der Energiequelle.

Die Ökonomie ist biophysikalisch, sie besteht also aus Energie- und Materialflüssen. Jede wirtschaftliche Aktivität erfordert den Einsatz von Ressourcen und Energie und produziert Abfall (zusätzlich zum gewünschten Produkt). Das bedeutet, dass eine absolute Entkopplung zwischen Wirtschaftswachstum und Umweltbelastung - ein weit verbreiteter Mythos - nicht möglich ist. Das bedeutet auch, dass uns Innovationen nur in einem begrenzten Ausmaß ıretten können. Auch die derzeit populäre Strategie einer "Kreislaufwirtschaft« hat Grenzen (Giampietro 2019). Der Begriff spielt auf eine auf biologischen Kreislaufprozessen basierende Wirtschaft an, mit der eine industrielle Kreislaufwirtschaft jedoch nur wenig zu tun hat. Da Materialien in kleinen Mengen nur schwer aus Produkten zurückgewonnen werden können, Recycling viel Energie erfordert und Materialien ab einem bestimmten Punkt ihre Eigenschaften verlieren können, ist ein reduzierter Energie- und Materialeinsatz der einzig gangbare Weg.

Die herrschende Umweltpolitik betont in der Regel die Bedeutung von Effizienzsteigerung und vermeidet das Thema des gesamtwirtschaftlichen Energie- oder Ressourcenverbrauchs. Zwar können Ressourcen in vielen Fällen immer noch effizienter genutzt werden, doch es gibt Grenzen der Effizienzsteigerung. Noch wichtiger ist die Tatsache, dass Effizienzverbesserungen ohne die Festlegung von Obergrenzen für den Ressourcen- und Energieverbrauch aufgrund von Rebound-Effekten nicht zu einem geringeren Ressour- 
cenverbrauch führen werden. Dieser Effekt wurde erstmals von Stanley Jevons beschrieben, der beobachtete, dass Verbesserungen der Kraftstoffeffizienz bei der Dampfmaschine zu einem erhöhten, und nicht zu einem geringeren Einsatz von Kohle führten.

Aufgrund ihres biophysikalischen Zugangs stehen die Grenzen des Wachstums im Zentrum des Ansatzes der ökologischen Ökonomie. In den letzten Jahren wurde dieser Ansatz zunehmend mit Erkenntnissen der kritischen politischen Ökonomie und der Degrowth-Literatur zusammengeführt. Davon ausgehend wurde sowohl der strukturelle Wachstumstrieb im Kapitalismus als auch dessen Abhängigkeit von Wachstum zur Verhinderung von Arbeitslosigkeit und damit verbundenen sozialen Unruhen diskutiert. Gleichzeitig gibt es aber auch eine soziale Dimension der Wachstumsökonomie, der traditionell wenig Aufmerksamkeit geschenkt wurde. Die übliche Erzählung (sowohl auf der Linken als auch auf der Rechten) lautet, dass Wirtschaftswachstum »die Armen aus der Armut holt«. Zunehmend wird jedoch erkannt, dass nicht alle >emporgehoben « werden und dass einige Gruppen tatsächlich einen hohen Preis für den Anstieg des materiellen Wohlstands anderer Gruppen bzw. andernorts bezahlen (siehe z.B. Kothari/Shrivastava 2012).

Diese Perspektive geht über Theorien ungleicher Entwicklung hinaus, auch wenn sie auf diesen aufbaut. Bereits in den 1970er Jahren formulierte Johan Galtung seine Theorie des strukturellen Imperialismus, mit der er zeigte, wie sowohl die Eliten als auch die Arbeiter:innenklasse in den Industrieländern von der Ausbeutung der sogenannten Entwicklungsländer profitierten, während die Armen bzw. die Arbeiter:innenklasse in den Entwicklungsländern am meisten verloren. In jüngerer Zeit haben Ulrich Brand und Markus Wissen (2017) das Phänomen mit dem Konzept der »imperialen Lebensweise» gefasst. Ein Aspekt dieser Entwicklung ist, dass sich Gewerkschaften immer stärker national orientierten, während die internationale Solidarität zusehends dahinschwand. Heute ist daher die internationale Bewegung für Umweltgerechtigkeit die wichtigste gesellschaftliche Kraft, die mit dem Konzept der »Klimagerechtigkeit « daran erinnert, dass der hohe und steigende Konsum im Globalen Norden und in den Mittel- und Oberschichten des Globalen Südens auf Kosten marginalisierter Länder und Bevölkerungsteile im Globalen Süden geht.

Das wirft die Frage auf, ob Wirtschaftswachstum gar nicht die Lösung für Armut ist, sondern Armut, Elend und Ungerechtigkeit vielmehr inhärent hervorbringt. Sollte dies der Fall sein, wäre das ein weiterer Grund, sich intensiv mit der Wachstumsproblematik zu beschäftigen. Vor allem die PostDevelopment-Literatur hat dargelegt, wie die Gleichsetzung von Entwicklung mit Wachstum in der Praxis einer imperialistischen Politik diente, die 
eher den reichen als den armen Ländern zugutekam (Sachs 1999/2015) ${ }^{6}$. Die Degrowth-Bewegung, die in den 1970er Jahren in Frankreich ihren Anfang nahm, verband die anthropologische Kritik an der Zerstörung von Kulturen andernorts mit einer kulturellen Kritik am Entwicklungsmodell in den westlichen Gesellschaften selbst (vgl. für eine gute Übersicht Muraca 2013). In diesem Sinne geht es nicht nur um den Globalen Süden und die Probleme, die dort im Zuge der Durchsetzung des westlichen Wachstumsmodells entstehen. Es geht vielmehr um eine Kritik der »Wachstumsgesellschaft« selbst, an der modernen Kultur und dem westlichen Entwicklungsmodell, das nach immer >mehrs strebt, eine Kritik der Akkumulation und der Kommodifizierung menschlicher Beziehungen.

All diese Gesichtspunkte müssen von den GND-Vorschlägen ernst genommen werden, wenn sie die vielfältigen Krisen, zu deren Überwindung sie antreten, tatsächlich bewältigen wollen. Das erfordert auch, die institutionellen Grundlagen der Wachstumsgesellschaft selbst zu verändern. Der folgende Abschnitt diskutiert daher, inwieweit die aktuellen GND-Vorschläge diesen tiefgreifenden Herausforderungen tatsächlich gerecht werden.

\section{Bewertung der Vorschläge für einen Green New Deal}

Aus den im vorherigen Abschnitt dargestellten Überlegungen folgt, dass wir den Energie- und Rohstoffverbrauch reduzieren ebenso wie eine Obergrenze für den Gesamtverbrauch dieser Ressourcen festlegen müssen. Eine noch radikalere Implikation ist die Notwendigkeit, unsere Wirtschaftssysteme so umzustrukturieren, dass ihr Funktionieren nicht mehr vom Wirtschaftswachstum abhängt. Ich werde mich im Folgenden sowohl mit der Frage befassen, inwieweit die Vorschläge für einen GND die im vorigen Abschnitt diskutierten Aspekte beleuchten, als auch - und das ist entscheidend - ob sie politische Maßnahmen enthalten, die radikal genug sind, um den zentralen Problemen der gegenwärtigen politischen Ökonomie in ihrem gesamten Ausmaß gerecht zu werden.

\section{Verringerung des Energie- und Materialverbrauchs}

Die Verwendung von Mainstream-Begriffen wie ssaubere` oder >grüne` Energie und Technologie ist in allen vier Vorschlägen für einen GND verbreitet. Auch die Energieeffizienz ist ein zentrales Thema, insbesondere bei der GND-

6 Für eine tiefergehende Analyse der vielfältigen problematischen Aspekte der Wachstumsökonomie einschließlich Militarisierung und Versicherheitlichung von Lieferketten, Wettbewerb, Hedonismus, Produktivismus und sogar Eugenik vgl. Spash (2020). 
Gruppe. Viel weniger thematisiert wird die Notwendigkeit oder das Ziel, den Gesamtenergieverbrauch zu reduzieren (meist geht es um fossile Brennstoffe, Kohlenstoffemissionen und Ungleichheit, die reduziert werden müssen).

Eine Ausnahme in dieser Hinsicht stellt der Vorschlag von DiEM dar, da hier die Notwendigkeit sowohl eines reduzierten Energieverbrauchs als auch einer Verringerung des Materialdurchsatzes anerkannt wird. Im Hinblick auf Energie wird dies meist unter dem Aspekt diskutiert, wie die Nachfrage nach Energie reduziert werden kann. Dabei geht DiEM davon aus, dass ihre politischen Projekte für integrierte Wohnungs-, Versorgungs- und Mobilitätsstrategien allein eine massive Verringerung des Energiebedarfs bewirken werden. DiEM schlägt jedoch auch ein Gesetz vor, das darauf abzielt, den Materialdurchsatz in Europas Lieferketten zu reduzieren - ein Vorschlag, der auf den ersten Blick viel mit dem Ansatz der Kreislaufwirtschaft gemeinsam hat. Interessanterweise sind sich die Autoren des Rebound-Effekts bewusst und schlagen eine (bewegliche) Obergrenze für den Materialeinsatz vor, um diesem entgegenzuwirken: »Dies könnte übergangsweise durch eine Deckelung des jährlichen Materialdurchsatzes unterstützt werden, die jedes Jahr verschärft werden könnte« (Adler u.a. 2019: 71). Angesichts dessen überrascht es, dass DiEM die neue EU-Taxonomie für soziale und grüne Investitionen unterstützt, die wegen ihres enormen Greenwashing-Potenzials kritisiert wird (Hache 2020).

\section{Ein wesentlicher Unterschied: Null-Emissionen vs. Netto-Null-Emissionsziele}

In engem Zusammenhang mit den Ambitionen in Bezug auf Energie- und Ressourcennutzung stehen die Ziele der verschiedenen GND-Vorschläge zur Verringerung von Treibhausgas bzw. $\mathrm{CO}_{2}$-Emissionen. Der Ausstieg aus fossilen Brennstoffen würde zu einem enormen Rückgang der $\mathrm{CO}_{2}$-Emissionen führen. Dennoch würde dies nicht ausreichen, um Null-Emissionen zu erreichen, da es noch einige andere bedeutende Emissionsquellen gibt (z.B. die Zementproduktion). Allerdings streben nur sehr wenige Länder oder Unternehmen tatsächlich an, ihre Emissionen auf null zu reduzieren - trotz des scheinbar ehrgeizigen Ziels »Netto-Null«, das sich in den letzten Jahren zunehmend verbreitet. Netto-Null-Emissionen anzustreben, bedeutet Emissionen zuzulassen, aber dafür zu sorgen, dass sie durch Offsetting, d.h den Kauf von Kompensationen, oder durch Negative-Emissionen-Technologien rausgeglichen w werden.

Offsetting bzw. Kompensationslösungen werden seit Langem von kritischen Wissenschaftler:innen und Umweltorganisationen kritisiert, sowohl wegen der fehlenden tatsächlichen Emissionsreduktion als auch wegen der negativen sozialen und ökologischen Auswirkungen, insbesondere auf die Biodiversität und im Globalen Süden, wo die meisten Kompensationsprojekte durchgeführt werden. Auf Netto-Null-Ziele zu setzen, kann sogar zu einem Anstieg 
der Emissionen führen, weil mit Kompensationen die Weiterverwendung fossiler Brennstoffe legitimiert wird, wobei die Kompensation gleichzeitig nur geringe Auswirkungen mit Blick auf die Entwicklung der $\mathrm{CO}_{2}$-Emissionen hat. Eine Koalition von Organisationen für Klimagerechtigkeit hat kürzlich einen Bericht veröffentlicht, der aufzeigt, wie die vorherrschenden Netto-Null-Ziele klimapolitische Untätigkeit verschleiern (Actionaid u.a. 2020).

In ähnlicher Weise ist die Annahme einer erfolgreichen und großflächigen Einführung von Negative-Emissionen-Technologien Teil der Klimastrategie vieler Länder, bis zu einem bestimmten Datum Netto-Null zu erreichen, und diese Annahme ist auch zentral für das Pariser Klimaabkommen (Spash 2016). Von Seiten der Klimawissenschaften wird jedoch darauf hingewiesen, dass es diese Technologien noch gar nicht gibt oder es unwahrscheinlich ist, dass sie in der erforderlichen Größenordnung und innerhalb des erforderlichen Zeithorizonts verfügbar sein werden. Viele anerkannte Klimawissenschaftler:innen sehen sie daher als >spekulativ an (vgl. etwa Anderson 2019).

Dennoch setzen sowohl DiEM als auch der Vorschlag der US-Demokrat:innen auf Netto-Null-Ziele. Während die US-Demokrat:innen dies offensichtlich unkritisch tun, warnt DiEM in einer Fußnote vor Offsets: "Ein gerechter Übergang kann nicht auf einer fortgesetzten Kompensation der Emissionen durch den Einsatz groß angelegter Dekarbonisierungsstrategien beruhen « (Adler u.a. 2019: 63). Im Gegensatz dazu ist der Labour-GND sehr ambitioniert und strebt sogar absolut null Emissionen bis 2030 an. Dies ist nicht nur äußerst ehrgeizig, sondern zeigt auch, dass sie die Bedeutung der Unterscheidung zwischen Null- und Netto-Null-Emissionen erkannt haben.

\section{Die Probleme des Wirtschaftswachstums}

In Bezug auf Wirtschaftswachstum reichen die vier GND-Vorschläge von dessen Befürwortung bis zu dessen Problematisierung. Während die USDemokrat:innen und die GND-Gruppe explizit herausstreichen, wie ihre Pläne zu Wirtschaftswachstum führen werden, verzichtet der Labour-GND auf den Begriff Wachstum (positioniert sich aber auch nicht wachstumskritisch). Eine explizite Problematisierung ist nur im DiEM-Vorschlag zu finden.

Die GND-Gruppe erkennt zwar die Existenz biophysikalischer Grenzen an. Im Vorwort ihres Berichts bezieht sie sich sogar auf den ökologischen Ökonomen Herman Daly und erklärt, dass »dieser Bericht, indem er einen Green New Deal vorschlägt, die Grenzen unseres Ökosystems anerkennt; dass >die Biosphäre endlich, nicht wachsend, geschlossen und durch die Gesetze der Thermodynamik eingeschränkt ist « (eig. Übersetzung). Diese Erkenntnis wird jedoch in dem Bericht nicht weiterverfolgt. Er stützt sich vielmehr stark auf die konventionelle (Klima-)Ökonomie und, wie bereits erwähnt, ins- 
besondere auf den Stern-Report - ein Bericht mit einer klaren Orientierung an Wirtschaftswachstum, der sich der Argumente für ein grünes Wachstums bedient und im Klimawandel neue Geschäftsmöglichkeiten erblickt. In jüngerer Zeit haben Stern und Kollegen vertreten, dass das Wirtschaftswachstum durch die Energiewende in Richtung einer »neuen Klimaökonomie« angekurbelt werden wird (GCEC 2014). ${ }^{7}$

Der Wachstumsfokus ist auch zentral für viele Ökonom:innen, die derzeit bei der sozialdemokratischen Linken beliebt sind, wie Ha-Joon Chang und Mariana Mazzucato. Chang (2011) hat zum Beispiel argumentiert, dass ein Problem des marktliberalen Kapitalismus darin bestehe, dass er das Wirtschaftswachstum verlangsamt. Ähnliche Argumente finden wir bei (Post-) Keynesianer:innen, die sich für soziale Absicherung, weniger Ungleichheit und mehr Inklusion einsetzen, weil dies zu höheren Raten des Wirtschaftswachstums führe. Mazzucato hat ihrerseits an politischen Empfehlungen für einen GND mitgewirkt, die sich auf nachhaltiges Wachstum konzentrieren und den Klimawandel zu einer Chance für Investitionen und Innovationen machen sollen (Mazzucato/McPherson 2018). Damit bedient sie sich einer Sprache, die viel eher dem grünen Wachstumsdiskurs entspricht als den neueren linken Debatten über einen GND. In dieser Hinsicht haben Mazzucato und Stern viele Gemeinsamkeiten.

Diese im weiteren Sinne problematischen Seiten des Wirtschaftswachstums werden jedoch in kaum einem der vier GNDs berücksichtigt. Dass die GNDVorschläge das gesamte Spektrum der Wachstumsprobleme nicht zur Kenntnis nehmen oder nicht diskutieren wollen, wird vielleicht am klarsten daran, dass sie Wachstum als systemisches Problem selbst gar nicht erst erwähnen. Zum Beispiel geht keiner der GND-Vorschläge darauf ein, dass unser gegenwärtiges Wirtschaftssystem auf kontinuierliches Wachstum angewiesen ist, um Arbeitslosigkeit und soziale Probleme zu verhindern, und ebenso wenig, dass die wesentliche Dynamik einer kapitalistischen Wirtschaft auf dem Akkumulationsdrang beruht. Vielmehr stützen sich die Vorschläge implizit auf irgendeine Vorstellung von einer veränderten Ausrichtung des Wachstums.

7 Die Global Commission on the Economy and Climate (GCEC), der inzwischen auch Lord Stern angehört, hat das Argument auf den Kopf gestellt: „Wenn der Klimawandel nicht angegangen wird, ist auf lange Sicht das Wachstum selbst gefährdet « (GCEC in Spash 2016). Das Vorzeigeprojekt des GCEC, die »neue Klimaökonomie« (New Climate Economy) wurde 2013 von den Regierungen von sieben Ländern in Auftrag gegeben, um »Regierungen, Unternehmen und der Gesellschaft zu helfen, besser informierte Entscheidungen darüber zu treffen, wie wirtschaftlicher Wohlstand und Entwicklung bei der Bewältigung des Klimawandels erreicht werden können « (eig. Übersetzung). Das Projekt spielte eine Schlüsselrolle bei der Vorbereitung des Pariser Klimaabkommens durch Publikationen mit vielsagenden Titeln wie »Better Growth, Better Climate« (GCEC 2014) und »Seizing the Global Opportunity» (GCEC 2015). 
DiEM zum Beispiel stellt explizit bestimmte Arten von Wachstum in Frage, wie extraktives oder grünes Wachstum. Doch obwohl sich alle GND-Vorschläge darum drücken, den Begriff „Entkopplung « zu verwenden, scheinen sie die Überzeugung zu teilen, dass es möglich ist, den Inhalt des Wachstums zu verändern - eine Position, die letztlich auf die Überzeugung hinausläuft, eine Entkopplung von Wirtschaftswachstum und Ressourcennutzung sei möglich.

Auf den ersten Blick mag es so aussehen, als ob DiEM die Probleme der Wachstumsgesellschaft grundlegender in Frage stellt - vor allem mit der achten Säule ihres GND »Schluss mit dem Dogma des endlosen Wachstums«. Der Begleittext erkennt die »Besessenheit vom Wirtschaftswachstum « als Haupttreiber der Klima- und Ökokrise an. Aber ist die »Besessenheit « von Wachstum oder das Wachstum selbst das Problem? DiEM konzentriert sich auf Ersteres. Es sind vor allem das BIP-Wachstum als übergeordnetes gesellschaftliches Ziel und die gescheiterte wachstumsorientierte Politik, die in Frage gestellt werden. Wie so oft in der populären wachstums-agnostischen Literatur (z.B. der sogenannten `Donut Ökonomie`) liegt der Fokus auf der Ebene der Ideen - so als ob es keine ökonomisch-strukturellen Zwänge gäbe. Die problematische Schlussfolgerung aus einer solchen Argumentation lautet, dass wir einfach unsere Indikatoren ändern können und die Probleme der Wachstumsgesellschaft damit verschwinden würden. Der Vorschlag von DiEM ist zwar der einzige, der einige der Probleme der Wachstumsgesellschaft berührt, aber er geht nicht weit genug: Eine grundlegende Infragestellung einer wachstumsbasierten Wirtschaft darf nicht mit der Infragestellung des BIP als allgemeinem Orientierungspunkt der Wirtschaftspolitik verwechselt werden.

\section{Das Problem des Produktivismus}

Die fehlende Infragestellung des Wachstums könnte durch den Einfluss eines keynesianischen Ansatzes erklärt werden, der für jeden New Deal zentral ist. Schon der Ausgangspunkt eines New Deal macht es schwer, Wachstum in Frage zu stellen, da der Produktivismus und der Einsatz >ungenutzter Ressourcen< (d.h. Arbeit) den Kern des Ansatzes ausmachen. Das zeigt sich etwa beim DiEM-Vorschlag, dessen zweite Säule genau darin besteht, ungenutzte Ressourcen wieder einzusetzen, oder genauer gesagt: »Ungenutzte EU-Ressourcen in öffentliche Dienstleistungen [zu] pumpen «(Adler/Wargan oJ.: 4). Der Bericht sorgt sich auch um die Produktivität, wenn Genossenschaften gefördert werden, weist aber darauf hin, dass diese die Arbeitsplatzsicherheit erhöhen, die Arbeitnehmer:innen stärken und »insgesamt mindestens so produktiv sind wie kapitalistische Unternehmen« (Adler u.a. 2019: 51). Der Labour-GND erwähnt die Krise des britischen Produktivitätsniveaus und schöpft aus den Arbeiten von Mazzucato neue Hoffnung. 
Mit der Post-Work-Literatur könnte man stattdessen fragen: Warum diese Obsession mit Vollbeschäftigung? Warum können die Menschen nicht den Luxus haben, mehr freie Zeit zu haben, untätig zu bleiben, ihre Zeit lieber für Gemeinschaftsaufgaben, Pflegearbeit, Kunst oder politische Arbeit einzusetzen? Sicherlich kann es der Fall sein, dass gesellschaftliches Arbeitsvermögen brachliegt, obwohl die Gesellschaft dieses Arbeitsvermögen sinnvoll einsetzen könnte. Aber die Diskussion wird in der Regel nicht so geführt. Warum fangen wir nicht mit der Frage an, was produziert werden muss, damit die Bürger ein gutes und sinnvolles Leben führen können? Wenn wir dort ansetzen, wäre schnell klar, dass mehr Arbeitsressourcen freigesetzt werden könnten, wenn wir die unnötige Produktion (etwa von fossilen Brennstoffen, Waffen, Autos, Luxusartikeln oder billigen und umweltschädlichen Konsumgütern) und all die unnötigen Aufgaben einstellen, die derzeit in der Arbeitswelt ausgeführt werden (sogenannte `Bullshit-Jobs`, vgl. Graeber 2018).

Angesichts des notwendigen Ausstiegs aus fossilen Brennstoffen und des notwendigen Übergangs zu einer weniger energieintensiven Wirtschaft werden wir in Zukunft jedoch mehr menschliche Arbeitskraft benötigen, um die gleichen Aufgaben zu erledigen. Das liegt daran, dass unsere so genannte Arbeitsproduktivität in Wirklichkeit eher darauf beruht, dass wir menschliche Arbeitskraft durch Maschinen und andere Energiequellen ersetzen, und nicht darauf, dass die Menschen selbst so viel intelligenter und effizienter arbeiten. Aus dieser Überlegung folgt, dass in Postwachstumsökonomien die Produktivität sinken muss (Mair u.a. 2020) - eine für den keynesiansichen Ansatz durchaus herausfordernde Schlussfolgerung.

\section{Soziale Dimension und internationale Solidarität}

Obwohl die soziale Dimension einen Schwerpunkt in allen GND-Vorschlägen darstellt, ist die Perspektive der Vorschläge überwiegend nationalstaatlich, d.h. sie konzentrieren sich auf den Abbau von Ungleichheit auf nationaler Ebene. Wo die internationale Dimension angesprochen wird, geht es meist um Finanz- oder Technologietransfers. Nicht thematisiert wird dabei das Thema der strukturellen Ungleichheit des Weltwirtschaftssystems. Selbst der Aspekt der internationalen Umweltgerechtigkeit wird kaum thematisiert, trotz der starken Betonung von Gerechtigkeit im Allgemeinen und der engen Verbindung des US-GND zur Umweltgerechtigkeits-Bewegung.

Abgesehen von den Umweltproblemen im Zusammenhang mit Wirtschaftswachstum sucht man in den GND-Vorschlägen auch vergeblich nach einer allgemeineren Kritik an der Wachstumsgesellschaft, insbesondere am so genannten Wachstumsimaginär, wie es in der Degrowth-Literatur diskutiert wird, d.h. unsere kulturelle Konditionierung, ständig `noch mehr zu 
wollen. Dementsprechend fehlt auch eine Auseinandersetzung sowohl mit der >Konsumgesellschaft « als solcher als auch mit den mittleren Konsumniveaus und -mustern und der Lebensweise im Globalen Norden. Vielmehr geht es um eine Verminderung der Einkommensungleicheit (und des Konsums?) sowie um "gut bezahlte, gewerkschaftlich organisierte Arbeitsplätze« (LabourGND) oder gar die »Anhebung des Lebensstandards « (Adler/Wargan o.J.: 10).

Obwohl es sowohl im Vorschlag von Labour als auch von DiEM um eine Transformation des Wirtschaftssystems geht, die über den Übergang von einer auf fossilen Brennstoffen basierenden zu einer auf erneuerbaren Energien basierenden Wirtschaft hinaus geht, stellen beide Projekte weder den mittleren Lebensstandard - oder gar den der Arbeiter:innenklasse - im globalen Norden noch dessen Abhängigkeit vom globalen Süden direkt in Frage. Die Vorschläge machen es sich in dieser Hinsicht einfach und legen den Schwerpunkt der Kritik auf die reichsten Teile der Gesellschaft, ganz so als ob der Lebensstandard oder das Konsumniveau anderer Klassen nicht problematisch wäre.

Zur Verteidigung von DiEM könnte man vorbringen, dass es DiEM nicht explizit um einen höheren materiellen Lebensstandard geht, sondern vielmehr darum, »allen Kommunen mehr Wohlstand, Sicherheit und Freiheit « zu bringen (Adler/Wargan o.J.: 11). Doch selbst wenn DiEM auf solch subtile Weise versucht, \Lebensstandard « neu zu definieren, ist diese Art der Darstellung des Themas unbefriedigend. Denn das Problem des westlichen Lebensstandards quer durch alle Klassen bleibt ausgeklammert - insbesondere, dass die moderne »imperiale Lebensweise« (Brand/Wissen 2017) auf einem unverhältnismäßig hohen Anteil am globalen Konsum basiert, sowohl im Hinblick auf Produkte als auch auf Ressourcen und Umweltsenken. Da das vorherrschende Modell wirtschaftlichen Wachstums in den Ländern des Globalen Nordens auf der Extraktion sowohl finanzieller als auch materieller Ressourcen aus dem Globalen Süden beruht, muss eine angemessene Auseinandersetzung mit diesem Thema weit über nationale Umverteilungspolitik hinausreichen und die Funktionsweise des Weltwirtschaftssystems grundlegender in Frage stellen.

\section{Green New Deals, grünes Wachstum und Konjunkturpakete nach der Pandemie}

Immer mehr -Staats- und Regierungschefs in der EU fordern, dass der European Green Deal in den Mittelpunkt des Aufbauplans der EU nach der Pandemie gestellt wird. Damit wird auch die Forderung verbunden, dass der Aufschwung nicht nur Wachstum und Arbeitsplätze schaffen, sondern auch ıgrün sein solle (Simon 2020). Obwohl der European Green Deal einige pro- 
gressive Elemente enthält, ist er in erster Linie ein grüner Wachstumsdeal ${ }^{8}$ und geht die oben dargestellten Probleme nicht an. Insofern ist von einem solchen grünen Aufbauplan in der EU wenig zu erwarten, solange er nicht weit über seinen ursprünglichen Inhalt hinausgeht.

Die Vorschläge für einen GND waren dazu gedacht, eine Wirtschafts- und Finanzkrise zu bewältigen, die bereits vor Corona vorhanden war. Obwohl das Coronavirus und die Eindämmungsmaßnahmen die wirtschaftliche Situation für einige Gruppen, die bereits zuvor nur schwer über die Runden kam, verschlechtert hat, müssen heute nicht unbedingt ganz andere Maßnahmen in Betracht gezogen werden als jene, die bereits vor der Pandemie diskutiert wurden. Entscheidend ist zunächst, die Vorstellung zu hinterfragen, dass >wirtschaftliche Erholung« Wirtschaftswachstum bedeuten muss. Es könnte stattdessen bedeuten, die Wirtschaft umfassend zu reorganisieren - und zwar so, dass ein größerer Teil der Produktion, der Ressourcennutzung und der Verteilung gesellschaftlich entschieden oder gesteuert wird, dass die Wirtschaft nicht in eine Krise gerät, wenn sie nicht wächst, und dass die Grundbedürfnisse der Menschen befriedigt werden können, ohne dass sie auf Lohnarbeit angewiesen sind.

Das kann sich natürlich nicht von heute auf morgen ändern. Was also tun mit der dominanten Wachstumsökonomie? Wie einen Kurswechsel einleiten, ohne gesellschaftliches Chaos hervorzurufen? Kurzfristig ließe sich die Nachfrage erhöhen, wobei sichergestellt werden muss, dass dies die Konsummöglichkeiten der ärmeren Bevölkerungsteile erhöht. Dies würde bedeuten, auf allgemeine expansive Maßnahmen wie die Senkung der Mehrwertsteuer oder andere Steuersenkungen zu verzichten, welche den Konsum aller Bevölkerungsgruppen ankurbeln. Expansive Maßnahmen wirken zwar schnell, würden aber lediglich die problematische Struktur der Wirtschaft bewahren. Stattdessen sollte auf Transfers an niedrige Einkommensgruppen gesetzt werden. Dies muss jedoch begleitet werden durch eine öffentliche Debatte über die Abhängigkeit des westlichen Konsumniveaus vom Globalen Süden und über einer Problematisierung der Konsumgesellschaft im Allgemeinen. Nur dann ist es möglich, Ansätze zu überwinden, die lediglich auf eine Umverteilung von oben nach unten abzielen, ohne das moderne Streben nach nie endendem materiellem Wachstum und Wohlstand in Frage zu stellen. Vor allem aber sollten die Regierungen sicherstellen, dass Menschen, die während der Pandemie ihre Arbeit und/oder ihr Einkommen verloren haben, in ihren Grundbedürfnissen wie Nahrung und Wohnung versorgt werden.

8 Die Europäische Kommission (2019) bezeichnet den Green Deal ausdrücklich als Europas »neue Wachstumsstrategie». 
Eine solche kurzfristig orientierte Politik muss durch eine langfristige Planung für eine Postwachstumsökonomie ergänzt werden. Diese müsste sowohl demokratisches Eigentum bzw. die Kontrolle über wichtige Bereiche der Produktion als auch die Bereitstellung einer allgemeinen Grundversorgung umfassen. Viele dieser Elemente sind bereits in den GND-Vorschlägen enthalten. Es gibt jedoch insbesondere zwei Aspekte in den aktuellen GNDEntwürfen, die neu bewertet werden müssen: der Fokus auf Wachstum und Produktivität und der Fokus auf Vollbeschäftigung bzw. die Schaffung von Arbeitsplätzen. Auf jeden Fall gilt es, wirtschaftliche Aktivitäten zu unterlassen, die zwar Arbeitsplätze schaffen, aber den Bedürfnissen der Gesellschaft und 'grünen`Zielen zuwiderlaufen. Das bedeutet nicht, dass die Ziele von fairen Jobs (US-GND), gewerkschaftlich organisierten Jobs (Labour-GND) und menschenwürdigen Arbeitsplätzen (DiEM) falsch sind. Dennoch muss vor allem die Linke es wagen, mit der Problematisierung von Arbeit in die Post-WorkDebatte einzusteigen, anstatt ihre ganze Energie auf Arbeitsplatzgarantien und das Recht auf Arbeit zu konzentrieren.

\section{Schlussfolgerungen}

Die in diesem Artikel vorgestellten und diskutierten Vorschläge für einen GND haben gemeinsam, dass sie den Klimawandel und die Notwendigkeit einer radikalen Transformation unserer Volkswirtschaften und Gesellschaften ernst nehmen. Dennoch scheint keiner der Vorschläge das gesamte Ausmaß der Herausforderung zu erkennen, die mit dem Übergang weg von fossilen Energiequellen mit hoher Energiedichte hin zu erneuerbaren Energien verbunden sind.

Es gibt zweifellos viele fortschrittliche und ehrgeizige Elemente in diesen Vorschlägen. Die Frage ist jedoch, ob sie angesichts der anstehenden Herausforderungen ehrgeizig genug sind. Das Ergebnis der hier vorgenommenen Bewertung lautet: nein. Die größte Schwäche ist die mangelnde Bereitschaft - oder das Zögern -, die Wachstumsökonomie direkt in Frage zu stellen. Natürlich könnte dahinter die strategische Überlegung stehen, dieses Thema nicht in den Vordergrund der Projekte zu stellen. Es könnte auch sein, dass die keynesianisch inspirierten Grundlagen der Vorschläge ein Hinausdenken über Wachstum und Produktivismus blockieren. Wenn wir Fragen der globalen Umweltgerechtigkeit aber wirklich angehen wollen, wenn wir die Hoffnung auf demokratische Entscheidungen, die den globalen Norden aus seiner ökologisch zerstörerischen Ökonomie und imperialen Lebens- und Funktionsweise herausführen, nicht aufgeben wollen, dann kommen wir um das Thema Wachstum nicht umhin. 
Darüber hinaus verschweigen alle Vorschläge die Tatsache, dass erneuerbare Energien weder wirklich >grün« noch ssauber s sind. Das Wort ısauber zu vermeiden, hätte hier helfen können, um die Einsicht zu vermitteln, dass auch erneuerbare Energien keine unproblematischen Lösungen sind. Die Verwendung solcher Etiketten ist besonders überraschend, wenn man bedenkt, dass einige der GND-Vorschläge den Anspruch haben, in ihrem Verständnis der ökologischen Krise nicht nur den Klimawandel zu berücksichtigen. Daher lässt sich insgesamt als eine zentrale Schlussfolgerung festhalten, dass die GND-Projekte aufgrund der fehlenden Auseinandersetzung mit grundlegenden Zusammenhängen der Thermodynamik eher sozial sind als ıgrün‘.

Es ließe sich auch eine Liste von Kritikpunkten aufstellen, die in den GNDVorschlägen fehlen, darunter die Kritik an der Wachstumsgesellschaft (dem Imaginären), an der Wachstumsabhängigkeit (dem Kapitalismus), am Konsumniveau im Globalen Norden (der Moderne) oder an der Konsumgesellschaft im weiteren Sinne. Auch fehlen Vorschläge, den Energie- und Ressourcenverbrauch einfach auf einem bestimmten Niveau zu deckeln (DiEM ist hier eine Ausnahme), und es fehlt eine Auseinandersetzung mit der Problematik der Ausbeutung von ohnehin marginalisierten Gruppen im globalen Süden durch den globalen Norden. Außerdem werden weder die gegenwärtigen Produktionsweisen und die Produktionsniveaus noch die Produktionsverhältnisse in Frage gestellt.

Dies führt zu einer Schlüsselfrage: Ist der GND lediglich ein Projekt zur Rettung des Kapitalismus? Das war eindeutig das ursprüngliche Ziel von Keynes mit seinen Vorschlägen für eine wirtschaftliche Erholung. Und da alle GND-Vorschläge explizit sowohl auf den New Deal als auch auf Keynes Bezug nehmen, könnte man leicht zu dem Schluss kommen, dass dies tatsächlich der Fall ist, zumal keiner der Vorschläge den Kapitalismus explizit in Frage stellt. Gleichzeitig weisen viele der Elemente, insbesondere in den Vorschlägen von DiEM und Labour, jedoch in eine postkapitalistische Richtung.

Insofern gibt es trotz der oben genannten Kritikpunkte und der Tatsache, dass die GNDs nicht weit genug gehen, tatsächlich viele Elemente, die in einer wirklich transformativen rot-grünen Strategie zum Tragen kommen sollten. Dazu gehören: die Bereitstellung einer allgemeinen Grundversorgung (außerhalb des Marktes); die Demokratisierung von Teilen der wirtschaftlichen Produktion; Vorschläge für alternative Wirtschaftsinstitutionen; (öffentliche) Investitionen in den öffentlichen Nahverkehr; der Fokus auf Gerechtigkeit und Ungleichheit sowie marginalisierte Bevölkerungsgruppen; das Ziel, aus fossiler Energie auszusteigen; das BIP durch andere Indikatoren für gesellschaftlichen Erfolg zu ersetzen sowie die Kritik am unregulierten Finanzmarktkapitalismus.

Eine tiefgreifende sozial-ökologische Transformation erfordert, Wirtschaft (wieder) als ein System der sozialen Versorgung zu betrachten. Wir müssen 
kollektiv definieren, wie wir unsere physischen Ressourcen gemeinsamen nutzen, nicht nur unsere Arbeitskraft, die traditionell im Fokus keynesianischer Ansätze steht. Aber wir müssen auch Arbeit neu betrachten und über die ausgeprägte Arbeitsethik hinausgehen, die in sozialdemokratischen und linken Parteien so verbreitet ist. Anstelle einer Arbeitsplatzgarantie und der Angewiesenheit von Menschen auf einen Arbeitsplatz zur Befriedigung ihrer Grundbedürfnisse sollten wir über andere Möglichkeiten der sozialen Organisation und andere Institutionen nachdenken. Die radikaleren GNDs, wie Labour und DiEM, bieten viele sinnvolle politische Vorschläge, die Teil von Strategien des Neuaufbaus nach der Corona-Pandemie und eines längerfristigen rot-grünen Transformationsprojektes sein könnten. Kombiniert mit Erkenntnissen aus der ökologischen Ökonomie, Degrowth und Post-Development sind sie von großer Bedeutung, um eine wirklich transformative Strategie zu entwerfen. Doch der Begriff New Deal ist zu stark in keynesianischen Produktivismus und Wachstum verstrickt - lassen wir ihn besser hinter uns.

Aus dem Englischen von Etienne Schneider

\section{Literatur}

Actionaid / Corporate Accountability / Friends of the Earth International / Global Campaign to Demand Climate Justice / Third World Network / What next? (2020): NOT ZERO: How snet zero> targets disguise climate inaction. Joint technical briefing by climate justice organisations.

Adler, David / Wargan, Pawel (o.J.): 10 Säulen des Green New Deals für Europa. The Green New Deal for Europe.

Adler, David / Wargan, Pawel / Prakash, Sona (Hg.) (2019): Roadmap. Für Europas sozial-ökologische Wende.The Green New Deal for Europe.

Anderson, Kevin (2019): Brief response to the UK Government's »net-zero« proposal. URL: https://kevinanderson.info/, Zugriff: 15.10.2019.

Brand, Ulrich (2014): Social ecological transformation: a crossover project between the left and the greens? URL: https://www.degrowth.info/, Zugriff: 1.12.2020.

Brand, Ulrich/ Wissen, Markus (2017): The imperial mode of living. In Spash, Clive L. (Hg.): Routledge Handbook of Ecological Economics. Nature and Society. Abingdon-New York:152-161.

Candeias, Mario (2013): Green Transformation. Competing strategic projects. Berlin.

Chang, Ha-Joon (2011): 23 things they don't tell you about capitalism. London.

Europäische Kommission (2019): Der europäische Grüne Deal. COM (2019) 640 final.

Galtung, Johan: A Structural Theory of Imperialism, In: Journal of Peace Research 8(2): 81-117.

GCEC (2014): Better Growth Better Climate. The New Climate Economy Report. Washington, D.C.

GCEC (2015): Seizing the Global Opportunity. The 2015 New Climate Economy Report. Washington, D.C.

Giampietro, Mario (2019): On the circular bioeconomy and decoupling: Implications for sustainable growth. In: Ecological Economics 162: 143-156. DOI: https://doi. org/10.1016/j.ecolecon.2019.05.001.

Graeber, David (2018): Bullshit Jobs: A Theory. London.

Hache, Frederic (2020): 50 Shades of Green. Sustainable Finance 2.0. The securitization of climate and biodiversity policies. Green Finance Observatory. Brüssel. 
Hoffmann, Maja / Paulsen, Roland (2020): Resolving the sjobs-environment-dilemmar? The case for critiques of work in sustainability research. In: Environmental Sociology 6(4): 343-354. DOI: https://doi.org/10.1080/23251042.2020.1790718.

Kothari, Ashish/Shrivastava, Aseem(2012): Why Is India Still Poor? In: Yes! Magazine, June 2012. Mair, Simon / Druckman, Angela / Jackson, Tim (2020): A tale of two utopias: Work in a postgrowth world. In: Ecological Economics 173: 1-11.

Mazzucato, Mariana / McPherson, Martha (2018): The Green New Deal: A bold mission-oriented approach. UCL: Institute for Innovation and Public Purpose.

Muraca, Barbara (2013): Décroissance: A Project for a Radical Transformation of Society. In: Environmental Values 22(2): 147-169.

Sachs, Wolfgang (2015): Planet dialectics: Explorations in environment and development. London. Simon, Frédéric (2020): France, Germany join group of $10 \mathrm{EU}$ countries calling for green recovery. URL: https://www.euractiv.com/, Zugriff: 19.1.2021.

Spash, Clive L. (2016):The political economy of the Paris Agreement on human induced climate change: a brief guide. In: Real-World Economics Review 75: 67-75.

- (2020): Apologists for growth: passive revolutionaries in a passive revolution. In: Globalizations. DOI: http://doi.org10.1080/14747731.2020.1824864.

- / Smith, Tone (2019): Of ecosystems and economics: re-connecting economics with reality. In: Real World Economics Review 87: 212-229.

Stern, Nicholas u.a. (2006): Stern Review: The Economics of Climate Change. London.

Taylor, Matthew (2019): Labour members launch Green New Deal inspired by US activists (22.3.2019). URL: www.theguardian.com, Zugriff: 22.3.2019.

The Green New Deal Group (2008): A Green New Deal. Joined-up policies to solve the triple crunch of the credit crisis, climate change and high oil prices: New Economics Foundation.

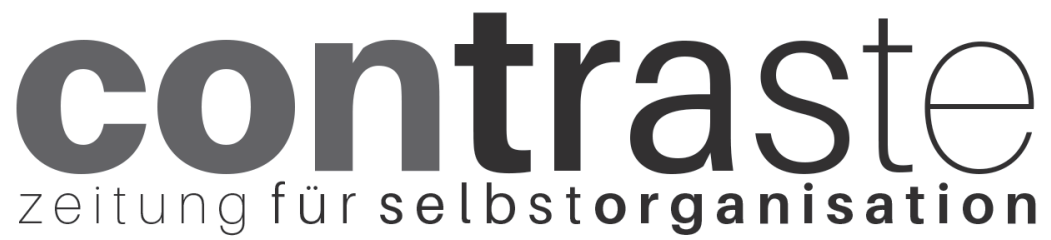

38. JAHRGANG

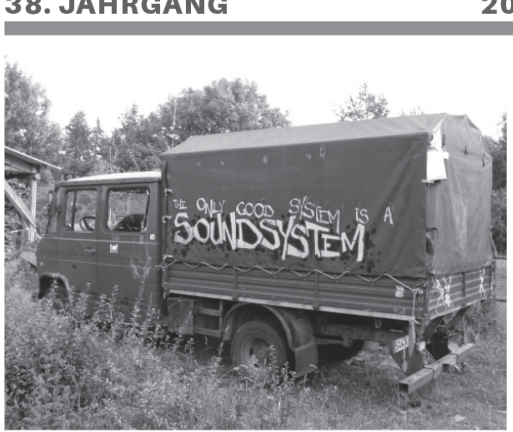

4'50 EUR

CONTRASTE, die einzige überregionale Monatszeitung für Selbstorganisation, dient seit über 35 Jahren den Alternativen Bewegungen als Sprachrohr und Diskussionsforum. AktivistInnen aus den unterschiedlichsten Bewegungen verfolgen mit der Herausgabe der Zeitung das Ziel, zu den von Globalisierung, Sozialabbau und Umweltzerstörung geprägten Verhältnissen Alternativen zu diskutieren, Entwicklungen aufzuzeigen, eigene Utopien zu entwickeln und diese zu erproben.

Gern versenden wir für $9 €$ ein dreimonatiges Schnupperabo.

\section{www.contraste.org}

